Reprod. Nutr. Dévelop., 1987, 27 (2 B), 441-459.

\title{
Ontogenèse des hormones de l'axe somatotrope
}

P. M. DUBOIS, Fiona J. HEMMING

C.N.R.S. UA 559, Faculté de Médecine Lyon-Sud, B.P. 12, 69600 Oullins, France.

\section{Summary. Ontogenesis of the somatotropic function.}

The ontogenesis of the somatotropic function involving its regulatory hormones is considered from an anatomical and functional point of view. Embryogenesis of the hypothalamo-pituitary unit involving development of the hypothalamo-hypophyseal portal system is complete during early fetal life as shown in a certain number of mammalian species. The pattern of development of animals is generally similar to that described in man. The maturation of the neuroendocrine mechanisms controlling the secretion of fetal pituitary growth hormone is analyzed in the present paper. Corticosteroids may act directly on the differentiation of the somatotropic cells. During fetal life, the plasma level of $\mathrm{GH}$ is always higher than after birth. Despite large gaps in our knowledge, SRIF may play a prominent part in the regulation of the somatotropic function, sometimes mediating the effect of other factors, and GRF may also be active during late gestation. However, maturation is not complete until after birth especially that of somatomedins as well as the pulsatile release of $\mathrm{GH}$.

\section{Introduction.}

Chez l'adulte, le fonctionnement des glandes endocrines se fait selon un système hiérarchisé impliquant généralement de nombreux facteurs qui peuvent agir soit sur les glandes endocrines périphériques elles-mêmes, soit sur les étages supérieurs du système, I'hypothalamus et l'antéhypophyse. Ainsi, les hormones hypothalamiques hypophysiotropes, dont la sécrétion est modulée par de nombreux facteurs périphériques ou centraux, rejoignent l'antéhypophyse en empruntant le système porte hypothalamo-hypophysaire. Les stimulines hypophysaires sont transportées par la circulation générale jusqu'aux glandes périphériques qu'elles contrôlent. Les glandes, à leur tour, peuvent agir sur leurs tissus-cibles. A chaque niveau, les différentes hormones agissent par l'intermédiaire de récepteurs. On a pu ainsi définir plusieurs axes à partir de l'appareil hypothalamohypophysaire : I'axe somatotrope, l'axe thyréotrope, l'axe gonadotrope, l'axe corticotrope.

L'axe somatotrope concerne l'hormone de croissance mais également les facteurs qui interviennent dans sa régulation. Chez l'adulte, l'action des facteurs hypothalamiques le régulant, la somatocrinine ou "Growth hormone 
releasing factor " (GRF) et la somatostatine (SRIF), est modulée par de nombreux facteurs. L'abondance de ces derniers rend difficile l'étude de leur maturation au cours du développement chez les animaux de laboratoire ou domestiques. Chez I'Homme, il se greffe des problèmes éthiques.

L'étude de la maturation de l'axe somatotrope implique un double aspect. Elle est, en effet, anatomique et fonctionnelle. Cette étude est encore compliquée par le facteur temps car la maturation commence pendant la vie embryonnaire puis fœtale, et se poursuit après la naissance. Si l'expérimentation est relativement facile chez l'animal après la naissance, elle est très difficile pendant la vie fotale, l'interprétation des observations et des expérimentations peut être biaisée. II apparaît alors difficile d'apprécier le degré d'immaturité de l'axe somatotrope dont nous allons essayer de suivre le développement anatomique et la maturation fonctionnelle.

\section{I. - Maturation anatomique de l'axe somatotrope}

L'ontogenèse des composants anatomiques de cet axe se fait selon un modèle assez comparable chez les mammifères (Lubberhuizen, 1931; Nelson, 1933 ; Harrison et Shyrock, 1940 ; Kingsbury et Roemer, 1940; House, 1943; Schechter, 1971 ; Vitums, 1977) bien que la durée de la gestation soit extrêmement variable (tabl. 1). Elle a été l'objet de nombreuses études ces dernières années, ce qui a permis de mieux apprécier les rôles respectifs des différents facteurs dans la mise en place de cette fonction.

TABLEAU 1

Durée de la gestation.

\begin{tabular}{ll}
\hline Vache & $280 \mathrm{j}(270-306)$ \\
Homme & $280 \mathrm{j}$ \\
Babouin & $187 \mathrm{j}$ \\
Brebis & $147 \mathrm{j}(140-155)$ \\
Porc & $116 \mathrm{j}(107-124)$ \\
Lapin & $32 \mathrm{j}$ \\
Rat & $21 \mathrm{j}$ \\
Souris & $20 \mathrm{j}$ \\
\hline
\end{tabular}

A. - Ontogenèse de l'antéhypophyse.

1. - Organogenèse de l'hypophyse. - La morphogenèse de l'hypophyse commence précocément. II est, en effet, possible de voir la première ébauche, sous la forme de la poche de Rathke, dès le stade de 7 paires de somites. Dans le temps, ce stade anatomique est atteint à des moments variables, selon l'espèce considérée. Cette première ébauche apparaît comme un diverticule du plafond du stomodeum ou bouche primitive. Elle est située en avant de la membrane pharyngienne. La poche de Rathke se développe en s'enfonçant dans le mésenchyme sus-jacent qui sépare le stomodeum des cavités encéphaliques 
(Atwell, 1926 ; Daikoku, 1958a, b ; Falin, 1961 ; Conklin, 1968 ; Andersen et al., 1971). Elle est constituée de deux feuillets aplatis dans le sens ventro-dorsal, feuillets séparés par la fente hypophysaire. Les deux feuillets vont șe développer de façon très inégale puisque le feuillet antérieur donnera le lobe antérieur de I'hypophyse, et le feuillet postérieur sera à l'origine du lobe intermédiaire.

Le lobe antérieur de l'hypophyse résulte du développement préférentiel du feuillet antérieur ou rostral de la poche de Rathke vers l'avant d'une part, et, d'autre part, latéralement. Ce mouvement latéral va isoler des cellules du mésenchyme pio-hypophysaire qui enveloppe l'ébauche, formant les fosses d'Atwell.

2. - Histogenèse de l'antéhypophyse. - Le lobe antérieur de l'hypophyse est constitué de cordons de cellules indifférenciées, de petite taille. Entre ces cordons de nombreux capillaires se développent (Atwell, 1926 ; Daikoku, 1958a, b ; Falin, 1961 ; Conklin, 1968 ; Andersen et al., 1971 ; Dubois, 1971a, b). Les cellules se différencient progressivement avec le développement des différents organites cellulaires : les ribosomes deviennent de plus en plus nombreux tandis que l'ergastoplasme ou réticulum endoplasmique rugueux est encore peu développé, ainsi que I'appareil de Golgi (Dumont et Dubois, 1967). L'ergastoplasme devient de plus en plus abondant et prend la disposition en sacs parallèles caractéristique de la cellule somatotrope différenciée (Dumont et Dubois, 1967). Le glycogène se raréfie dans la cellule (Dubois, 1971a). L'appareil de Golgi se développe : chaque dictyosome devient de plus en plus complexe (Dubois, 1972), tout en établissant des relations avec les dictyosomes voisins. Le système des lysosomes se met en place (Dubois et Tachon, 1974). Les grains de sécrétion sont visibles. Il existe des cellules différenciées dans l'antéhypophyse (Maillard, 1963 ; Dubois et Dumont, 1965, 1966 ; Dubois, 1968 ; Yoshimura et al., 1970 ; Schechter, 1971 ; Fink et Smith, 1971 ; Svalander, 1974 ; Hemming et al., 1983, 1986).

3. - Les cellules différenciées somatotropes et autres. - On peut en effet décrire plusieurs types de cellules, chacun avec des caractéristiques précises. Les méthodes classiques faisant appel à l'histochimie ou à la microscopie électronique n'ont apporté qu'une aide très limitée (Daikoku, 1958 ; Falin, 1961 ; Dubois et Dumont, 1965, 1966 ; Conklin, 1968 ; Dubois, 1968, 1971). On a pu décrire les cellules orangeophiles ou PAS + (Magre et Dupouy, 1973). Mais ce que I'on met en évidence n'est qu'une affinité tinctoriale ou un radical chimique. La microscopie électronique elle-même ne permet souvent de raisonner que par analogie avec ce que l'on peut observer chez l'adulte (Maillard, 1963 ; Dubois et Dumont, 1965, 1966 ; Dubois, 1968, 1971; Yoshimura et al., 1970; Fink et Smith, 1971; Schechter, 1971 ; Svalander, 1974). L'identification fonctionnelle précise est difficile à réaliser sans faire appel à des techniques telles que l'immunocytochimie qui, associées aux dosages radioimmunologiques ont permis d'avancer rapidement dans la connaissance de l'ontogenèse de l'hypophyse, à condition cependant d'en contrôler la spécificité et de les rendre suffisamment sensibles, ce qui est particulièrement important pour l'étude des stades précoces.

Ces méthodes ont, en effet, montré que l'hormone somatotrope apparaît au niveau de l'hypophyse à des moments extrêmement variables de la gestation 
selon les espèces (tabl. 2) (Stokes et Boda, 1968 ; Dubois, 1971 ; Baker et Jaffe, 1975 ; Setalo et Nakane, 1976 ; Bégeot et al., 1977 ; Chatelain et al., 1979).

TABLEAU 2

Apparition de l'hormone somatotrope.

\begin{tabular}{|c|c|c|c|}
\hline & $\begin{array}{l}\text { Rappel durée } \\
\text { de la gestation }\end{array}$ & Hypophyse & Sang \\
\hline Bovins & $280 \mathrm{j}$ & $\begin{array}{c}\text { ge semaine } \\
\text { ic (Dubois et al., 1971) }\end{array}$ & \\
\hline Homme & $280 \mathrm{j}$ & $\begin{array}{l}\text { 8e semaine } \\
\text { IC (Bégeot et al., 1977) } \\
\text { RIA (Matsuzaki et al., 1971) }\end{array}$ & $\begin{array}{c}68 \mathrm{j} \\
\text { (Kaplan et al., 1972) }\end{array}$ \\
\hline Rhésus & $166 j$ & & $\begin{array}{c}90 \mathrm{j} \\
\text { (Holland } \text { et al., 1979) }\end{array}$ \\
\hline Ovins & $147 j$ & $\begin{array}{c}60 \mathrm{j} \\
\text { IC (Stockes et Boda, 1968) } \\
\text { RIA (Charrier, 1973) }\end{array}$ & $\begin{array}{c}60 \mathrm{j} \\
\text { (Bassett } \text { et al., 1970) }\end{array}$ \\
\hline Rat & $21 \mathrm{j}$ & $\begin{array}{l}19 \mathrm{i} \\
\text { IC (Setalo et Nakane, 1976) } \\
\text { RIA (Rieutort, 1974) }\end{array}$ & $\begin{array}{c}19 \mathrm{j} \\
\text { (Rieutort, 1972) }\end{array}$ \\
\hline Souris & $20 j$ & $18 j$ & \\
\hline
\end{tabular}

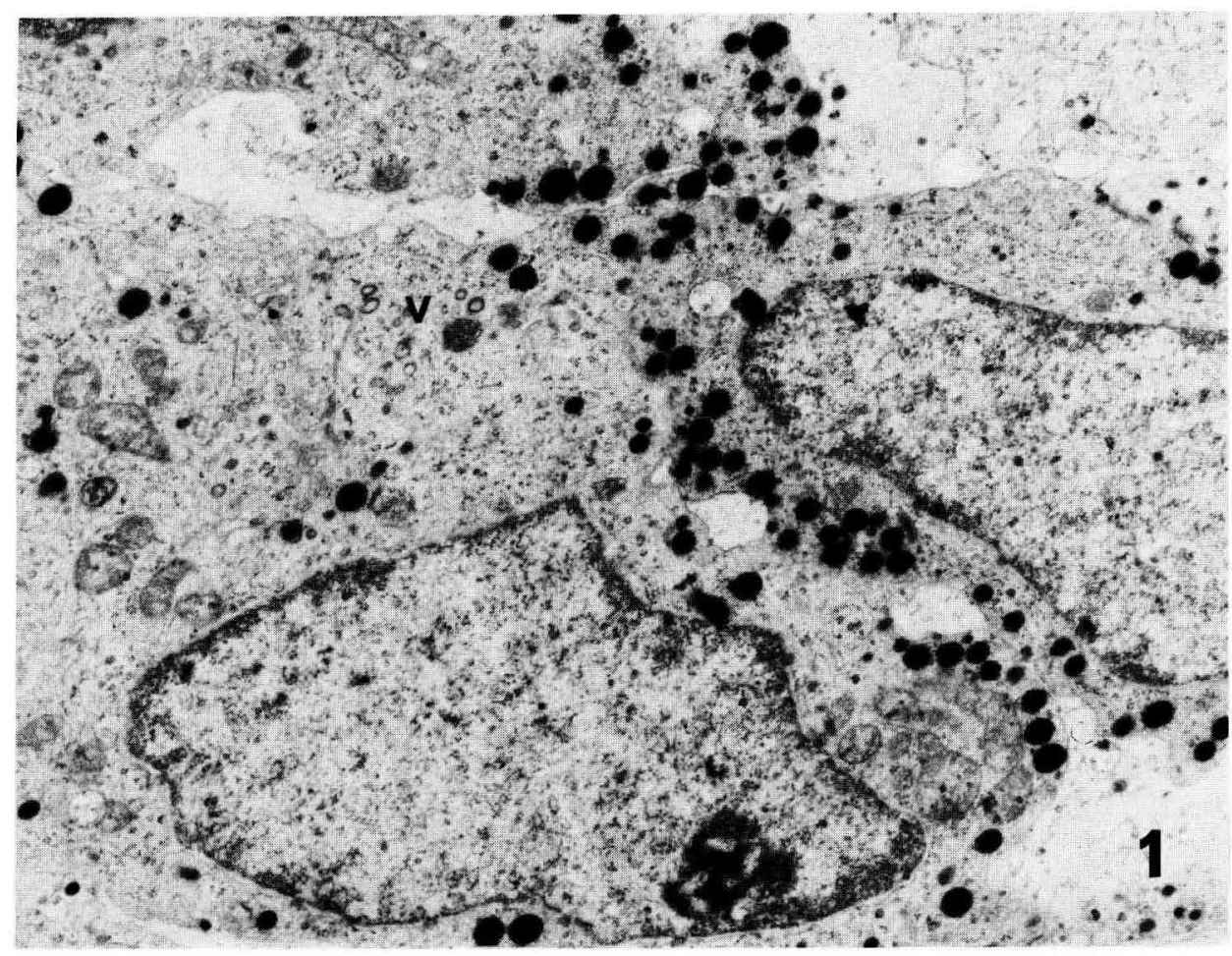

FIG. 1. - Cellules somatotropes mises en évidence dans l'hypophyse d'un foetus de rat à 20 jours de gestation. Remarquer les gros grains de sécrétion et l'abondance de vésicules hérissées \{v\}. $\times 36500$. 
Les cellules somatotropes peuvent également être identifiées en microscopie électronique par immunocytochimie chez le fotus humain (Li et al., 197)). Chez le Rat, l'immunocytochimie en microscopie électronique permet leur identification : à 19 jours, les grains de sécrétion sont encore peu nombreux. A 20 jours, les cellules somatotropes apparaissent groupées en petits amas de 3 ou 4 cellules. A ce moment, leur contenu en grains s'est accru (Hemming et al., 1986) (fig. 1).

\section{B. - Ontogenèse de l'hypothalamus.}

1. - Organogenèse. - En regard de la poche de Rathke, et un peu plus tard dans le temps, il apparaît une différenciation située au niveau du plancher du diencéphale. De là se forme un diverticule, l'infundibulum, qui descend à la rencontre de la poche, mais en restant toujours dans une position caudale. C'est de là que dérivent le lobe nerveux ou postérieur de l'hypophyse, la tige infundibulaire, l'éminence médiane, les noyaux de I'hypothalamus (O'Rahilly et Gardner, 1971).

2. - Histogenèse. - Chez l'Homme, les premiers noyaux hypothalamiques repérables, les noyaux préoptiques et prémamillaires sont visibles dès 6 semaines de gestation. La plupart des noyaux sont en place à la fin du 1er trimestre (Gilbert, 1935 ; O'Rahilly et Gardner, 1971). Ces formations sont susceptibles d'élaborer des amines (Hyyppa, 1972 ; Nobin et Bjorklund, 1973) et des peptides hypophysiotropes très précocément.

Chez le Rat, les noyaux se différencient à partir du $15^{\mathrm{e}}$ jour (Daikoku et al., 1971 ; Fink et Smith, 1971 ; Monroe et Paull, 1974 ; Pilgrim, 1974). Les facteurs hypothalamiques hypophysiotropes sont décelables parfois précocément. A ce sujet, il convient de faire remarquer que la sensibilité des méthodes utilisées peut jouer un rôle important ainsi que le suggère l'exemple de la gonadolibérine dont la présence fut d'abord signalée à 5 jours après la naissance (Araki et al., 1975) puis à 15 jours de gestation (Chiappa et Fink, 1977) mais qui peut, en fait, être dosée, dans I'encéphale certes, dès 12 jours de gestation (Aubert et al., 1985). Au sujet des formations aminergiques, l'emploi de l'immunocytochimie, plus sensible que la méthode de fluorescence induite, permet de mieux cerner le moment de l'apparition des amines. Ainsi récemment, Lidov et Mollivier (1982) d'une part, Wallace et Lauder (1983) d'autre part, ont montré par immunocytochimie que les fibres sérotoninergiques, en provenance des complexes B4 à B9 atteignent l'hypothalamus dès le jour 14 de la gestation.

3. - Les peptides hypothalamiques hypophysiotropes. - Les peptides hypothalamiques hypophysiotropes qui, chez l'adulte, participent à la régulation du fonctionnement de l'axe somatotrope apparaissent à des moments très variables. Le GRF apparaît tardivement. Cependant, chez l'Homme, son moment d'apparition varie selon les auteurs, entre 18 semaines (Bresson et al., 1984) et 28 semaines (Bloch et al., 1983). A 31 semaines, les fibres à GRF s'observent dans l'éminence médiane ; à la naissance, elles sont très nombreuses (Bloch et al., 1983). 
Chez le Rat, les neurones à GRF apparaissent à 19,5 jours de gestation (Daikoku et al., 1985) (tabl. 3).

\section{TABLEAU 3}

Differenciation de l'hypothalamus chez le rat.

Différenciation des noyaux hypothalamiques à partir du $15^{\mathrm{e}}$ jour chez le Rat (Fink et Smith, 1971).

\begin{tabular}{ll}
\hline LRF & $12 \mathrm{j}$ (Aubert et al., 1985) \\
TRF & $16 \mathrm{j}$ (Oliver et al., 1980) \\
SRIF & $17 \mathrm{j}$ (Mueller et al., 1978) \\
CRF & $18 \mathrm{j}$ (Bugnon et al., 1982) \\
GRF & $19 \mathrm{j}$ (Daikoku et al., 1985) \\
Dopamine, Adrenaline & $20 \mathrm{j}$ (Hyyppa, 1969) \\
\hline
\end{tabular}

La somatostatine est décelable chez l'Homme dès 10 semaines par dosage radioimmunologique (Kaplan et al., 1976). Les fibres immunoréactives apparaissent entre 13 et 16 semaines dans I'hypothalamus (Paulin et al., 1976 ; Bugnon et al., 1977). Chez le Rat, les premiers neurones à somatostatine s'observent dès 16 jours de gestation (Hoffman et al., 1980 ; Adachi et al., 1984 ; Pelletier et al., 1982).

\section{C. - Ontogenèse des connexions anatomiques hypothalamo-hypophysaires.}

Si l'on compare ces données avec celles déjà connues concernant la différenciation de l'antéhypophyse, on peut s'interroger sur l'éventualité de l'existence de relations fonctionnelles entre I'hypothalamus et I'hypophyse, ce qui sous-entend l'existence de relations anatomiques. On a pu mettre en évidence l'apparition précoce des vaisseaux du système porte chez diverses espèces. Chez la Souris, les capillaires du "Mantel plexus " existent dès 15 jours de gestation, à 16 jours les vaisseaux portes s'observent, le plexus primaire est visible dans les 5 premiers jours après la naissance (Enemar, 1961; Dearden et Holmes, 1976). Chez le Lapin, les éléments du système porte hypothalamo-hypophysaire se mettent en place entre 17 et 22 jours de gestation (Campbell, 1966 ; Terneby, 1972), entre 25 et 40 jours chez le Cobaye (Donovan et Peddie, 1973) ; chez le Cheval entre 24 et 49 jours (Vitums, 1977). Chez le Rat, les premières connexions vasculaires entre l'ébauche de l'éminence médiane et celle de l'antéhypophyse existent à 12 jours de gestation (Szabo et Czanyi, 1982). Les premiers vaisseaux portes apparaissent à 13 jours; les capillaires de l'antéhypophyse sont visibles à 15 jours; le "Mantel plexus " l'est à 15 jours. La zone palissadique de l'éminence médiane présente à 16 jours un aspect similaire à celui de l'adulte. Chez l'Homme, les connexions vasculaires hypothalamo-hypophysaires sont morphologiquement différenciées dès la $12^{\mathrm{e}}$ semaine et le système porte est fonctionnel (Thliveris et Currie, 1980). Il est à noter d'ailleurs que les premières fibres immunoréactives avec un anticorps dirigé contre un peptide hypothalamique hypophysiotrope, sont visibles généralement dans l'éminence médiane au niveau de leur terminaison au voisinage des capillaires, ce qui évoque bien sûr la possibilité d'une liaison fonctionnelle (Paulin et al., 1976, 1977 ; Bugnon et al., 1977). De plus, du fait de la 
texture très lâche des tissus embryonnaires aux stades précoces du développement, il n'est pas interdit de penser que des échanges puissent se faire entre les 2 parties de cet appareil hypothalamo-hypophysaire par simple diffusion.

\section{II. - Maturation fonctionnelle de l'axe somatotrope}

L'étude de la maturation de la régulation de la sécrétion de $\mathrm{GH}$ au cours de l'ontogenèse amène à s'interroger sur le rôle de ces facteurs de régulation, non seulement après, mais également avant la différenciation des cellules somatotropes.

\section{A. - La différenciation des cellules somatotropes.}

II a été admis jusqu'à ces dernières années que la différenciation des cellules antéhypophysaires était autonome, c'est-à-dire qu'elle se faisait indépendamment de l'hypothalamus, comme le suggèrent les études effectuées chez les fœtus humains anencéphales (Bégeot et al., 1977) ou après encéphalectomie chez le fœtus de Rat (Jost, 1953). L'étude de la différenciation des cellules somatotropes montre que cette notion d'autonomie est obsolète.

1. - L'anencéphalie chez l'Homme et l'encéphalectomie chez l'animal. Chez les fœtus humains anencéphales, il y a seulement une masse de tissu conjonctivo-vasculaire à la place du cerveau (Lemire et al., 1978); même par immunocytochimie, des neurones immunoréactifs n'ont jamais été trouvés à ce niveau. L'antéhypophyse est toujours présente (Lemire et al., 1978), les cellules somatotropes aussi, mais leur développement est perturbé comme le suggère l'étude de l'évolution de leur taille au cours de la gestation (Bégeot et al., 1977). Le taux sérique de GH est fortement diminué (Kaplan et al., 1972 ; Grumbach et Kaplan, 1973). Chez le Rat, l'encéphalectomie à 19,5 jours de gestation provoque essentiellement une diminution du taux plasmatique de $\mathrm{GH}$ (Rieutort et Jost, 1976). Ceci suggère que la différenciation des cellules somatotropes pourrait être autonome, ou tout au moins, indépendante de l'hypothalamus à défaut d'une autonomie vraie, tandis que l'activité sécrétoire et excrétoire serait sous un contrôle dans lequel l'hypothalamus jouerait un rôle important. 'Cependant, si l'hypothalamus est toujours absent dans le cas d'anencéphalie vraie, les autres glandes périphériques sont, elles, toujours présentes, même si elles présentent des modifications (Lemire et al., 1978). De plus, le GRF apparaît tardivement dans l'hypothalamus chez l'Homme (Bloch et al., 1983) ; chez le Rat son apparition est concomittante de celle des cellules somatotropes (Daikoku et al., 1985). Ceci suggère que I'hypothalamus pourrait ne pas intervenir dans la différenciation des cellules somatotropes.

2. - Etude expérimentale. - Pour apprécier l'influence des facteurs intervenant sur la différenciation des cellules antéhypophysaires, il est nécessaire d'explorer la période qui s'écoule entre l'apparition de l'ébauche et celle des premières cellules différenciées. La méthode de culture in vitro d'ébauche d'hypophyse de fœetus de rat développée par Watanabe et Daikoku (1976) permet 
ce type d'investigation, surtout lorsque le milieu de culture utilisé est entièrement synthétique (Bégeot et al., 1983).

La nature du milieu de culture est, en effet, importante puisque en présence de sérum de foetus de veau, quel que soit le jour de mise en culture, il n'y a pas de différenciation de cellules somatotropes. Le GRF n'est pas impliqué dans la différenciation mais il permet d'obtenir des cellules d'une taille plus grande. Ce sont en fait des facteurs périphériques qui interviennent, à condition que la dose d'insuline soit faible. Les corticostéroïdes semblent particulièrement importants (Hemming et al., 1984). Le rôle exact des autres facteurs ( $T_{3}$, glucagon) est encore en cours d'étude.

Ainsi chez le Rat, il est vraisemblable que les glucocorticoïdes jouent un rôle essentiel, mais associés à d'autres facteurs périphériques et à des facteurs de croissance. De toute façon les glucocorticoïdes existent chez le fœtus puisqu'on peut les doser dans le plasma (Dupouy et al., 1975) de même que les hormones thyroïdiennes qui à la naissance existent en quantité non négligeable (Dussault et Labrie, 1975). L'insuline et le glucagon sont également présents précocément (Girard et al., 1975). II est probable que dans le cas de nos cultures d'ébauche, l'insuline agisse soit par l'intermédiaire de ses propres récepteurs, soit par le biais des récepteurs des somatomédines (Oppenheimer et al., 1983 ; Van Wyk, 1985).

La différenciation des cellules somatotropes ne serait donc pas autonome mais serait sous la dépendance de divers facteurs, d'origine probablement périphérique. Les facteurs hypothalamiques eux-mêmes, le GRF notamment, ne semblent jouer qu'un rôle discret. Des facteurs placentaires peuvent intervenir puisque le placenta semble riche en facteurs, qui, au moins sur le plan immunologique ont un comportement voisin de celui des facteurs hypothalamiques hypophysiotropes même s'il n'y a pas avec eux d'identité chimique complète (tabl. 4).

\section{TABLEAU 4}

Neuropeptides détectés dans le placenta.

\begin{tabular}{ll}
\hline Homme $\left\langle{ }^{*}\right\rangle$ & TRH (Mitnik et al., 1974) \\
& TSH (Gibbons et al., 1975) \\
& GnRH (Mitnik et al., 1974) \\
& SRIF (Fitz-Patrick et Patel, 1981) \\
& CRF (Liotta et al., 1977) \\
& TRH (Shambaugh et al., 1983) \\
Rat & GnRH (Macaron et al., 1976) \\
& GRF (Baird et al., 1985) \\
Cobaye & TRH probablement (Nogimori et al., 1985) \\
& TRH hypothalamique $\neq$ TRH placentaire \\
& (Youngblood et al., 1980)
\end{tabular}

$\left({ }^{*}\right)$ Seuls sont cités ici les peptides ressemblant aux peptides hypothalamiques (voir revue de Simpson et McDonald, 1981).

Le moment de la mise en culture joue également puisque après mise en culture a 13 jours, il y a moins de cellules somatotropes au terme de la culture que si l'explantation est faite à 14 jours (Hemming et al., 1984). Comme la mise en culture a 12 jours ne permet pas d'obtenir des cellules somatotropes, il est vraisem- 
blable que les facteurs impliqués dans leur différenciation doivent agir au jour 13 et surtout au jour 14 de gestation.

Ces facteurs de différenciation agissent donc précocément. Le processus de différenciation une fois amorcé se poursuivra selon un programme précis. In vitro ou in vivo, les cellules somatotropes différenciées seront visibles au même moment, c'est-à-dire à 19 jours de gestation. Une partie seulement des cellules de l'ébauche mise en culture remplira ce programme, ce qui laisse supposer que les autres cellules sont déjà programmées pour donner d'autres types cellulaires.

\section{B. - GH au cours de l'ontogenèse.}

Chez le fœutus humain, la $\mathrm{GH}$ est présente à la $8^{8}$ semaine de gestation (Matsuzaki et al., 1971 ; Kaplan et al., 1972 ; Pavlova et al., 1968 ; Bégeot et al., 1977). Le contenu de l'antéhypophyse s'accroît pendant la gestation. La concentration se stabilise dans la $2^{\mathrm{e}}$ moitié de la gestation (Kaplan et al., 1972, 1976). Par contre, le taux sérique après une courte période d'élévation, puis une phase en plateau, diminue pendant la $2^{e}$ moitié de la gestation. Ceci suggère une prise de contrôle par un facteur inhibiteur (Kaplan et al., 1976). Chez le Mouton, pendant la vie fœetale, le taux sérique de $\mathrm{GH}$, toujours très élevé (Bassett et al., 1970), présente un aspect biphasique (Gluckman et al., 1979a, b). Chez le Rat, le contenu hypophysaire s'accroît pendant la vie fœtale et jusqu'à l'âge de 20 jours (Birge et al., 1967 ; Rieutort, 1974 ; Walker et al., 1977). Le taux plasmatique s'élève jusqu'au pic observé au moment de la naissance pour décroître ensuite dans les dix premiers jours avant de se stabiliser (Rieutort, 1974 ; Blazquez et al., 1974 ; Strosser et Mialhe, 1975). Au moment de la puberté une élévation progressive de $\mathrm{GH}$ apparaît à nouveau chez la femelle avec des variations importantes pendant le cycle œestral (Ojeda et Jameson, 1977).

Dans toutes les espèces étudiées, Homme, Rat, Mouton, Porc (Spencer et al., 1985), les taux sériques de $\mathrm{GH}$ sont toujours très élevés pendant la période fœtale. II semblerait que l'hormone somatotrope ainsi excrétée en grande quantité, le soit essentiellement sous forme de monomères (Gluckman et al., 1979a et b).

Les cellules somatotropes paraissent donc très actives. Elles synthétisent I'hormone somatotrope et l'excrètent dans le sang suivant des modalités très variables selon les espèces, imposant à l'esprit une prise de contrôle par les facteurs (hypothalamiques). Le niveau très élevé de $\mathrm{GH}$ dans le sang nous amène à nous interroger sur le pourquoi de ce phénomène. Est-il dû à des modalités particulières dans le mécanisme de différenciation des cellules somatotropes ? Est-il dû à un excès de facteurs inhibiteurs ? Peut-être encore est-il dû à une hypersensibilité des cellules somatotropes aux facteurs stimulants, ou inversement à un défaut de réceptivité de ces cellules aux facteurs inhibiteurs ? II est encore impossible de répondre à ces questions.

\section{C. - La régulation des cellules somatotropes.}

Comme chez l'adulte, deux facteurs hypothalamiques hypophysiotropes sont les agents essentiels de cette régulation, mais ils voient leur action modulée par de nombreux facteurs d'origine diverse. 
1. - Le GRF. - Des cellules antéhypophysaires fœtales maintenues en culture pendant 4 jours sont susceptibles de réagir au GRF par une augmentation de la sécrétion de GH (Baird et al., 1984). L'antéhypophyse fotale parait même être plus sensible puisque $40 \%$ des cellules seraient susceptibles de réagir. Cependant il convient dans l'interprétation de ces résultats de tenir compte de la durée de la culture, soit 4 jours. Ne doit-on pas alors considérer que les cellules étudiées doivent se comporter comme des cellules plus âgées?

En fait, plusieurs équipes ont montré que les cellules somatotropes en culture pouvaient réagir au GRF pendant la vie fœtale. Chez les rats plus âgés on peut constater que la sensibilité au GRF est beaucoup plus forte chez les jeunes rats qu'après le sevrage (Cella et al., 1985). De plus le contenu hypophysaire en GH est accru par un traitement de 5 jours avec le GRF (Cella et al., 1985). Le GRF entraîne, chez les fœtus de Mouton cathétérisés, une élévation de GH aussi bien à la mi-gestation qu'en fin de gestation, la réaction étant toujours plus forte chez les individus les plus jeunes (Ohmura et al., 1984). Chez le fotus humain, le GRF peut accroitre l'activité des cellules somatotropes dans des hypophyses prélevées entre 9 et 11 semaines et cultivées pendant plusieurs semaines. La quantité d'hormone somatotrope sécrétée s'accroît avec la dose de GRF (Goodyer et al., 1984).

Ainsi les cellules somatotropes seraient susceptibles de réagir au GRF pendant la vie fœtale, et, chez le sujet jeune avec une réactivité spectaculaire par rapport à celle observée chez les sujets plus âgés, c'est-à-dire à la fin de la gestation chez le Mouton ou après 20 jours chez le Raton. A quoi cela est-il dû ? Peut-être à la présence d'un grand nombre de récepteurs au GRF ? En fait, rien n'est encore connu à ce sujet. Si récepteur il y $a$, il conviendra de résoudre le problème de l'origine du GRF : le placenta (Baird et al., 1985), I'hypothalamus du fotus (Bloch et al., 1983) ou encore la mère. Une autre possibilité est une moindre réactivité aux facteurs inhibiteurs notamment à la somatostatine.

2. - La somatostatine. - Les cellules somatotropes fotales humaines maintenues in vitro sont susceptibles de réagir à l'adjonction de somatostatine au milieu de culture (Paulin et al., 1976; Marcovitz et al., 1982).

Dans un système de périfusion, la somatostatine n'a aucun effet sur la sécrétion de GH chez le Rat jusqu'à 4 jours après la naissance (Rieutort, 1981). Une absence de réactivité à la somatostatine a également été notée après incubation, pendant $3 \mathrm{~h}$, d'hypophyses de fœtus de 18 à 21 jours (Khorram et al., 1983). Une inhibition de la sécrétion n'apparaît qu'à 5 jours après la naissance. De même, l'injection in vivo de sérum anti-somatostatine à des fotus depuis le $15^{\mathrm{e}}$ jour de gestation ou à des nouveau-nés n'entraîne pas de modification du taux de $\mathrm{GH}$ avant la fin de la première journée après la naissance (Oliver et al., 1982). Par contre, à partir du $2^{\mathrm{e}}$ jour de vie, cette immunisation passive contre la somatostatine entraîne une élévation de la GH sérique (Oliver et al., 1982). Mais les cellules somatotropes du fœtus de Rat mises en culture à 18 jours et cultivées pendant $24 \mathrm{~h}$ réagissent à la somatostatine (Frawley et al., 1985). Il est à noter que chez le Rat ce n'est qu'après la naissance que le contenu hypothalamique en somatosta- 
tine s'accroît notablement et qu'il y a une corrélation entre la concentration de somatostatine hypothalamique et le taux sérique de GH (Walker et al., 1977).

Dans d'autres espèces telles que le Mouton (Gluckman et al., 1979a, b) et le Porc (Spencer et al., 1985), les fœtus portant un cathéter sont susceptibles de réagir à l'injection de somatostatine par une diminution du taux de $\mathrm{GH}$ plasmatique. Cependant dans le cas du Porc la réactivité est variable selon les fœtus quelques jours avant la mise bas (Spencer et al., 1985). Chez le Mouton à la migestation, il y a une diminution du taux sérique de GH (Gluckman et al., 1979). Peut-être est-ce dû à l'influence de la somatostatine ? Cela impliquerait la présence de récepteurs dont on suppose seulement l'existence.

3. - Les facteurs modulateurs. - Le GRF et la somatostatine sont connus pour avoir des effets directs sur les cellules somatotropes aussi bien in vivo qu'in vitro. Un certain nombre d'autres facteurs interviennent dans la régulation de $\mathrm{GH}$ mais le mécanisme de leur action est malaisé à appréhender pendant la vie fœtale. IIs peuvent agir au niveau hypothalamique en bloquant la sécrétion de somatostatine ; ils apparaissent alors comme des facteurs stimulants de la sécrétion de $\mathrm{GH}$.

Mais si le mécanisme de régulation de la sécrétion de GH par la somatostatine se met en place après la naissance comme chez le Rat, comment expliquer l'action stimulante du TRF à 20 jours de gestation dans un système de périfusion (Rieutort, 1981), ou encore à 21 jours (Khorram et al., 1983). Le TRF chez le Rat est d'apparition très précoce (Tonooka et Greer, 1978 ; Olivier et al., 1981). Y a-til, par un mécanisme d'existence transitoire, un effet direct du TRF sur les cellules somatotropes ; effet qui disparaîtrait lorsque le mécanisme normal impliquant la somatostatine se mettrait en place ? La réactivité des cellules somatotropes à la somatostatine n'apparaît-elle pas en fait plus précocément pendant la vie fœtale chez le Rat comme le suggèrent les travaux de Frawley et al. (1985) ? Peut-être s'agit-il simplement d'une particularité liée à l'espèce ? Ainsi chez l'Homme adulte, le TRF est sans effet sur la sécrétion de GH sauf chez les acromégales (Borges et al., 1983). Mais pendant la vie fœtale chez l'Homme, l'action du TRF est encore mal documentée. Chez le fœetus de Porc, par contre, la somatostatine semble impliquée à la fin de la période fœtale, et l'action stimulante du TRF sur I'hypophyse du fœetus cathétérisé, par rapport aux sujets témoins, pourrait peutêtre s'expliquer par ce mécanisme (Spencer et al., 1985).

Chez le Mouton, la sérotonine (5-HT) semble impliquée précocément puisque son administration a un effet stimulant sur la sécrétion de $\mathrm{GH}$, effet d'autant plus important que le fœtus cathétérisé est plus jeune (Marti-Henneberg et al., 1986). Cet effet est très probablement médié par l'hypothalamus puisque la section de la tige empêche tout effet de la 5-HT. Là encore comment interpréter cette diminution de l'effet stimulant en fonction de l'âge : s'agit-il d'une diminution du nombre de récepteurs à la sérotonine dans l'hypothalamus avec l'approche de la mise bas ? On peut admettre aussi l'immaturité du système impliquant la somatostatine.

La $\beta$-endorphine aurait un effet stimulant plus important avant 110 jours de gestation chez le Mouton soit par une action inhibitrice sur les neurones dopami- 
nergiques (Gluckman et al., 1980), soit par un autre mécanisme indirect impliquant la stimulation de GRF mais ceci reste à démontrer.

Peut-être est-ce en augmentant la sécrétion de somatostatine que l'apomorphine, un agoniste de la dopamine, agirait puisqu'elle diminue la sécrétion de $\mathrm{GH}$ chez le fœtus de Mouton (Gluckman et al., 1981).

L'action du stress est contradictoire : chez l'Homme, il pourrait avoir, pour certains, une action stimulante sur la sécrétion de GH (Aubert et al., 1971) ; pour d'autres, cette action du stress n'existe pas (Von Mulhendahl et al., 1976). Chez le Rat il semble que le stress à l'éther entraîne des réponses variables selon l'âge (Strbak et al., 1985) : une inhibition de la sécrétion de $\mathrm{GH}$ pouvant survenir après 1 jour seulement mais de façon transitoire puisqu'on ne la retrouve plus entre 12 et 21 jours alors qu'elle réapparaît ensuite. Cette action serait médiée par la somatostatine. Les anesthésiques ont une action variable après leur administration à la mère. Cette variabilité est fonction de la nature de l'anesthésique et de la durée de son application (Rieutort et Jost, 1976).

Ainsi il n'est pas toujours aisé d'expliquer de façon précise le mode d'action des différents facteurs qui interviennent dans la régulation de la sécrétion de $\mathrm{GH}$ par les cellules somatotropes au cours de l'ontogenèse, soit parce que les données restent encore fragmentaires, soit parce que la maturation des mécanismes hypothalamiques de régulation est encore incomplète avec des variations selon les espèces. Le fœtus de Mouton semble acquérir précocément un niveau suffisant de maturité des mécanismes centraux. Le Porc l'acquiert plus tardivement. Le Rat verrait, en effet se mettre en place ses systèmes de régulation dans les jours suivant la naissance (Wehrenberg, 1986). Quant à l'Homme, il semble avoir bien besoin de sa petite enfance pour « mûrir».

4. - Les somatomédines. - Malgré l'immaturité plus ou moins prononcée de son système de régulation, à quoi peut servir la GH ? II est classique de considérer qu'elle sert à la croissance, non pas seule, mais au moins avec la participation d'autres hormones comme les hormones thyroïdiennes. Mais ce rôle lui est contesté. S'il existe, il peut se faire soit directement soit par l'intermédiaire des somatomédines (SM). Mais pendant la vie fœtale le type prédominant de SM n'est pas le même que chez l'adulte: chez le fœus de Rat domine la "Multiplication-stimulating activity " (MSA) identique à l'" Insulin-like growth factor " (IGF)-II ou SMA (Moses et al., 1980 ; Adams et al., 1983a, b) ; chez le fœtus de Mouton, I'IGF-I peu abondante pendant la vie fœtale, augmente à la naissance tandis que I'IGF-II a une évolution inverse (Gluckman et Butler, 1983). Pendant la vie fœtale les somatomédines sont élaborées dans divers organes in situ (D'Ercole et Underwood, 1981). In vitro, les fibroblastes issus du poumon fœtal élaborent IGF-II puis IGF-I (Adams et al., 1983). La GH ne stimule pas la synthèse d'IGF-II (Adams et al., 1983) ce que confirment les travaux récents de Richman et al. (1985) qui utilisent des hépatocytes de fœtus de Rat. S'agit-il là encore d'immaturité des mécanismes centraux de régulation de l'hormone dite de croissance?

5. - Sécrétion pulsatile. - Cette immaturité des mécanismes de la sécrétion de $\mathrm{GH}$ se retrouve au niveau de la pulsatilité de la sécrétion. Chez le fœtus de 
Mouton, il semble que la sécrétion de $\mathrm{GH}$ soit de nature pulsatile dès 70 jours de gestation, mais avec une amplitude des "pulses » très importante, traduisant ainsi l'immaturité du système pendant la vie fœtale, immaturité dont serait responsable la somatostatine (Gluckman, 1984). Chez I'Homme et chez le Rat, il est admis que la sécrétion n'apparaît qu'après la naissance : à 3 mois chez I'Homme (Rusak et Zucker, 1979). Le rat cependant pourrait voir ses performances dans ce domaine s'améliorer puisque la concentration de sérotonine hypothalamique varierait rythmiquement dès 19 jours (Banky, 1984). De plus il pourrait y avoir une influence maternelle sur les rythmes fœtaux chez le Rat (Reppert et Schwartz, 1984). Cette influence maternelle pallierait l'immaturité des systèmes neuronaux chez le fœetus.

Ainsi l'axe somatotrope se différencie assez précocément pendant la vie fœtale. Il semble fonctionner selon des modalités particulières que l'on qualifie d'immatures, par référence au modèle adulte, alors que ce terme n'est peut-être que le masque de notre ignorance actuelle.

$12^{e}$ Réunion du groupe Développement INRA Montpellier, 28-30 mai 1986.

Remerciements. - Les auteurs remercient Madame M. Tschora pour son aide dans la préparation du manuscrit.

\section{Références}

ADACHI T., OHTSUKA M., HISANO S., TSURUO Y., DAIKOKU S., 1984. Ontogenetic appearance of somatostatin-containing nerve terminals in the median eminence of rats. Cell Tissue Res., 236, 47-65.

ADAMS S. A., NISSLEY S. P., GREENSTEIN L. A., YANG Y. W. H., RECHLER M. M., $1983 a$. Synthesis of multiplication-stimulating activity (rat insulin-like growth factor II) by rat embryo fibroblasts. Endocrinology, 12, 976-987.

ADAMS S. A., NISSLEY S. P., HANDWERGER S., RECHLER M. M., $1983 b$. Developmental patterns of insulin-like growth factor-I and II synthesis and regulation in rat fibroblasts. Nature, 302, 150-153.

ANDERSEN H., VON BULOW F. A., MOLLGARD K., 1971. The early development of the pars distalis of human foetal pituitary gland. Z. Anat. Entwickl. Gesch., 135, 117.138.

ARAKI S., TORAN-ALLERAND C. D., FERIN M., VAN DE WIELE R. C., 1975. Immunoreactive gonadotropin-releasing hormone $(\mathrm{GnRH})$ during maturation in the rat. Ontogeny of regional hypothalamic differences. Endocrinology, 97, 693-697.

ATWELL W. J., 1926. The development of the hypophysis cerebri in man, with special reference to the pars tuberalis. Am. J. Anat., 37, 159-194.

AUBERT M. L., BÉGEOT M., WINIGER B. P., MOREL G., SIZONENKO P. C., DUBOIS P. M., 1985. Ontogeny of hypothalamic $\mathrm{GnRH}$ and pituitary $\mathrm{GnRH}$ receptors in fetal neonatal rats. Endocrinology, 116, 1565-1577.

AUBERT M. L., SISTEK J., CHABOT V., BOSSART H., 1971. L'hormone de croissance hypophysaire foetale pendant l'accouchement. Schweig Med. Wochenschr., 101, 1102-1107.

BAIRD A., WEHRENBERG W. B., BOHLEN P., LING N., 1985. Immuno-reactive and biologically active growth hormone-releasing factor in the rat placenta. Endocrinology, 117. 1598 1601.

BAIRD A., WERHENBERG W. B., LING N., 1984. Ontogeny of the response to growth hormonereleasing factor. Reg. Peptides, 10, 23-28.

BAKER B. L., JAFFE R. B., 1975. The genesis of cell types in the adenohypophysis of the human fetus as observed with immunocytochemistry. Am. J. Anat., 143, 137-162. 
BANKY S., 1984. Diurnal fluctuations in hypothalamic serotonin concentration preceed the onset of the plasma corticosterone rhythm during ontogenesis of the rat. Neuroendocrinol. Lett., 6 , 207-212.

BASSETT J. M., THORBURN G. D., WALLACE A. L. C., 1970. The plasma growth hormone concentration of the fetal lamb. J. Endocrinol., 48, 251-257.

BEGEOT M., DUBOIS M. P., DUBOIS P. M., 1977. Growth hormone and ACTH in the pituitary of normal and anencephalic human fetuses: immunocytochemical evidence for hypothalamic influences during development. Neuroendocrinology, 24, 208-220.

BEEGET M., LI J. Y., DUBOIS M. P., DUBOIS P. M., 1983. Organ culture of the anterior pituitary in a synthetic medium. An immunocytochemical study. Acta endocrinol., 102, 35-41.

BIRGE C. A., PEAKE G. T., MARIZ I. K., DAUGHADAY W. H., 1967. Radioimmunoassayable growth hormone in the rat pituitary gland : effects of age, sex and hormonal state. Endocrinology, 81, 195-204.

BLAZQUEZ E., SIMON F. A., BLAZQUEZ M., FOA P. P., 1974. Changes in serum growth hormone levels from fetal to adult age in the rat. Proc. soc. exp. Biol. Med., 147, 780-783.

BLOCH B., BRAZEAU P., LING N., BOHLEN P., ESCH F., WEHRENBERG W. B., BENOIT R., BLOOM F., GUILLEMIN R., 1983. Immunohistochemical detection of growth hormone releasing factor in brain. Nature, 263, 251-257.

BORGES J. L. C., VSKAVITCH D. R., KAISER D. L., CRONIN M. J., EVANS W. J., THORNER M. O., 1983. Human pancreatic growth hormone-releasing factor-40 (hpGRF-40) allows stimulation of $\mathrm{GH}$ release by TRH. Endocrinology, 113, 1519-1521.

BRESSON J. L., CLAVEQUIN M. C., FELLMANN D., BUGNON C., 1984 . Ontogeny of the neuroglandular system revealed with hpGRF 44 antibodies in human hypothalamus. Neuroendocrinology, 39, 68-73.

BUGNON C., FELLMANN D., BLOCH B., 1977. Immunocytochemical study of the ontogenesis of the hypothalamic somatostatin-containing neurons in the human fetus. Cell Tissue Res., 183, 319-328.

BUGNON C., FELLMANN D., GOUGET A., CARDOT J., 1982. Etude immunocytochimique de l'ontogenèse du système neuroglandulaire à CRF chez le Rat. C. R. Acad. Sci. (Paris), 294. 599-604.

CAMPBELL H. T., 1966. The development of the primary portal plexus in the median eminence of the rabbit. J. Anat., 100, 381-395.

CELla S. C., LOCATEllI V., GENNARO V. de, PUgGiONI R., PINTOR C., MULleR E. E., 1985. Human pancreatic growth hormone $(\mathrm{GH})$-releasing hormone stimulates $\mathrm{GH}$ synthesis and release in infant rats. An in vivo study. Endocrinologv, 116, 574-577.

CHARRIER J., 1973. Evolution foetale et postnatale du contenu en hormone de croissance de I'hypophyse ovine. Ann. Biol. anim. Biochim. Biophys., 13, 155-163.

CHATELAIN A., DUPOUY J. P., DUBOIS M. P., 1979. Ontogenesis of cells producing polypeptide hormones (ACTH, MSH, LPH, GH, prolactin) in the fetal hypophysis of the rat : influence of the hypothalamus. Cell Tissue Res., 196, 409-427.

CHIIAPPA S. A., FINK G., 1977. Releasing factor and hormonal changes in the hypothalamuspituitary-gonadotrophin and adrenocorticotrophin systems before and after birth and puberty in male, female and androgenized female rats. J. Endocrinol., 72, 211-224.

CONKLIN J. C., 1968. The development of the human fetal adenohypophysis. Anat. Rec., 160, 79-92.

DAIKOKU S., 1958 a. Studies on the human foetal pituitary. I. Quantitative observations. Tokushima J. exp. Med., 5, 200-213.

DAIKOKU S., $1958 \mathrm{~b}$. Studies on the human foetal pituitary. II. On the form and histological development especially that of the anterior pituitary. Tokushima J. exp. Med., 5, 214-231.

DAIKOKU S., KAWANO H., NOGUCHI M., TOKUZEN M., CHIHARA K., SAITO H., SHIBASAKI T., 1985. Ontogenic appearance of immunoreactive GRF-containing neurons in rat hypothalamus. Cell Tissue Res., 242, 511-518.

DAIKOKU S., KOTSU T., HASHIMOTO M., 1971. Electron microscopic observations on the development of the median eminence in perinatal rats. Z. Anat. Entwicklung Gesch., 134, 311-322.

DEARDEN N. M., HOLMES R. L., 1976. Cyto-differentiation and portal vascular development in the mouse adenohypophysis. J. Anat., 121, 551-565. 
D'ERCOLE A. J., UNDERWOOD L. E., 1981. Growth factors in fetal growth and development, 155-182. In : NOVY M. J., RESKO J. A., Fetal endocrinology, Acad. Press, New York.

DONOVAN B. T., PEDDIE M. J., 1973. The development of the hypophysial portal system in the guinea pig. J. Anat., 114, 292-304.

DUBOIS M. P., 1971. Mise en evidence par immunofluorescence des cellules somatotropes et des cellules à prolactine dans l'hypophyse foetale des bovins. C. R. Acad. Sci. (Paris), 272. 433-435.

DUBOIS P., 1968. Données ultrastructurales sur l'antéhypophyse d'un embryon humain à la huitième semaine de son développement. C. R. Soc. Biol., 162, 689-692.

DUBOIS P., 1971 a. Le glycogène au cours de la différenciation cellulaire dans l'antéhypophyse foetale humaine. Z. Anat. Entwickl. Gesch., 133, 318-324.

DUBOIS P., $1971 \mathrm{~b}$. Les modifications de l'ultrastructure des capillaires et les variations de leurs rapports avec les cellules glandulaires durant l'histogenèse de l'antéhypophyse humaine. Z. Anat. Entwickl Gesch., 135, 242-252.

DUBOIS P., 1971. Signification fonctionnelle d'une catégorie cellulaire de l'antéhypophyse foetale humaine. C. R. Acad. Sci. (Paris), 273 D. 880-882.

DUBOIS P., 1972. Origine et développement de I'appareil de Golgi au cours de la différenciation cellulaire dans une glande endocrine chez l'Homme, I'antéhypophyse foetale. J. Microscopie, 13, 193-206.

DUBOIS P. M., BEGEOT M., AUBERT M. L., HEMMING F., MOREL G., 1984. Ontogenesis of pituitary hormones. In F. LABRIE, L. PROULX, Endocrinology, Elsevier, Int. Congr., Series 655, pp. 693-697.

DUBOIS P., DUMONT L., 1965. Observations en microscopie électronique du lobe antérieur de I'hypophyse embryonnaire humaine au troisième mois de la vie intra-utérine. C. $R$. Soc. Biol., 159, 1574-1576.

DUBOIS P., DUMONT L., 1966. Nouvelles observations au microscope électronique sur l'antéhypohyse humaine du troisième au cinquième mois du développement embryonnaire. $C$. R. Soc. Biol., 161, 2105-2107.

DUBOIS P., TACHON G. L., 1974. "GERL-complex » Iysosomes et grains de sécrétion au cours de la différenciation dans une glande endocrine chez l'Homme: I'antéhypophyse foetale. J. Microscopie, 19, 253-264.

DUMONT L., DUBOIS P., 1967. Quelques aspects ultrastructuraux de l'antéhypophyse foetale humaine. Rev. Iyon. Med., 16, 593-606.

DUPOUY J. P., COFFIGNY H., MAGRE S., 1975. Maternal and fetal corticosterone levels during late pregnancy in rats. J. Endocrinol., 65, 347-353.

DUSSAULT J. H., LABRIE F., 1975. Development of the hypothalamic-pituitary-thyroid axis in the neonatal rat. Endocrinology, 97, 1321-1324.

ENEMAR A., 1961. The structure and development of the hypophysial portal system in the laboratory mouse, with particular regard to the primary plexus. Ark. Zool., 13, 203-221.

FALIN L. I., 1961. The developement of the human hypophysis and differentiation of cells of its anterior lobe during embryonic life. Acta anat, 44, 188-205.

FINK G., SMITH G. C., 1971. Ultrastructural features of the developing hypothalamo-hypophyseal axis in the rat. A correlative study. Z. Zellforsch. 119, 208-226.

FITZ-PATRICK D., PATEL Y., 1981. Evidence of somatostatin precursors in human stomach, placenta and amniotic fluid. J. clin. Endocrinol. Metab., 53, 372-376.

FRAWLEY L. S., HOEFFLER J. P., BOOCKFOR F. R., 1985. Functional maturation of somatotropes in fetal rat pituitaries : analysis by reverse hemolytic plaque assay. Endocrinology, 116. 2355-2360.

GIBBONS J. M., MITNICK M., CHIEFFO V., 1975. In vitro biosynthesis of TSH- and LH-releasing factors by human placenta. Am. J. Obst. Gynecol., 121, 127-135.

GILBERT M. S., 1935. The early development of the human diencephalon. J. comp. Neurol., 62, $81-115$.

GIRARD J. R., KERVRAN A., ASSAN R., 1975. Functional maturation of the A ceil in the rat, 57-71. In : CAMERIANI-DAVAKIS R. A., COLE H. S., Early diabetes in early life, Acad. Press, New York.

GOODYER C., MARCOVITZ S., BEREZUIK M., DE STEPHANO L., LEFEBVRE Y., 1984. In vitro 
modulation of $\mathrm{GH}$ secretion from early gestation human pituitaries, 209-218. In : ELLENDORF F., GLUCKMAN P. D., PARVIZI N., Fetal neuroendocrinology, Perinatology Press, N. Y. GLUCKMAN P. D., 1984. Maturation of neuroendocrine control of pituitary hormone secretion, studies in the ovine fetus. In : F. LABRIE, L. PROULX, Endocrinology. Elsevier, Int. Congr., Series 655, 697-700.

GLUCKMAN P. D., BUTLER J. H., 1983. Parturition-related changes in insulin-like growth factors-I and $I I$ in the perinatal lamb. J. Endocrinol., 99, 223-232.

GLUCKMAN P. D., GRUMBACH M. M., KAPLAN S. L., 1981. The neuroendocrine regulation and function of growth hormone and prolactin in the mammalian fetus. Endocr. Rev., 2, 363-395.

GLUCKMAN P. D., MARTI-HENNEBERG C., KAPLAN S. L., LI C. H., GRUMBACH M. M., 1980. Hormone ontogeny in the ovine fetus. $X$. The effects of $\beta$-endorphin and naloxone on circulating growth hormone, prolactin and chorionic somatomammotropin. Endocrinology, 107, 76 80.

GLUCKMAN P. D., MUELLER P. L., KAPLAN S. L., RUDOLPH A. M., GRUMBACH M. M., 1979 a. Hormone ontogeny in the ovine fetus. I. Circulating growth hormone in mid and late gestation. Endocrinology, 104, 162-168.

GLUCKMAN P. D., MUELLER P. L., KAPLAN S. L., RUDOLPH A. M., GRUMBACH M. M., $1979 b$. Hormone ontogeny in the ovine fetus. III. The effect of exogenous somatostatin. Endocrinology, 104, 974-978.

GRUMBACH M. M., KAPLAN S. L., 1973. Ontogenesis of growth hormone, insulin, prolactin and gonadotropin secretion in the human fetus, 462-489. In Foetal and neonatal physiology. Proc. Sir Joseph Barcroft Centenary Symp. Cambridge. Cambridge Univ. Press.

HARRISON B. M., SHYROCK E. H., 1940. Cytogenesis of the pars distalis of the horse. Anat. Rec., 72, 449-471.

HEMMING F. J., BÉGEOT M., DUBOIS M. P., DUBOIS P. M., 1983. Ultrastructural identification of corticotropes of the fetal rat. In-vivo and in-vitro immunocytochemistry. Cell Tissue Res., $234,427-437$.

HEMMING F. J., BEEGEOT M., DUBOIS M. P., DUBOIS P. M., 1984. Fetal rat somatotropes in vitro : effects of insulin, cortisol and growth hormone-releasing factor on their differentia tion : a light and electron microscopic study. Endocrinology, 114, 2107-2113.

HEMMING F. J., DUBOIS M. P., DUBOIS P. M., 1986. Somatotrophs and lactotrophs in the anterior pituitary of fetal and neonatal rats. Electron microscopic in immunocytochemical identification. Cel/ Tissue Res., 245, 457-460.

HOFFMAN G. E., DICK L. B., GASH P., 1980. Development of somatostatin neurons : examination by the technique of combined autoradiography and immunocytochemistry. Peptides, 1 (Suppl. 1), 79-83.

HOLLAND F. J., SHERWOOD W. G., DIBATTISTA D., CUNA L., 1979. The ontogeny of growth hormone and prolactin in the rhesus monkey fetus. Proc. 61 st annu. Meet. Endocr. Soc., Anaheim, p. 222 (Abs).

HOUSE E. L., 1943. The development of the hypophysis of the ox. Am. J. Anat., 73, 1-25.

HYYPPA M., 1969. A histochemical study of the primary catecholamines in the hypothalamic neurons in the rat in relation to the ontogenetic and sexual differentiation. Z. Zellforsch. Mikrosk. Anat., 98, 550-561.

HYYPPA M., 1972. Hypothalamic monoamines in human fetuses. Neuroendocrinology, 9, $257-$ 266.

JOST A., 1953. Problems of fetal endocrinology: the gonadal and hypophyseal hormones. Rec. Progr. Horm. Res., 8, 379-418.

KAPLAN S. L., GRUMBACH M. M., AUBERT M. L., 1976. The ontogenesis of pituitary hormones and hypothalamic factors in the human fetus : maturation of central nervous system regulation of anterior pituitary function. Rec. Progr. Horm. Res., 32, 161-243.

KAPLAN S. L., GRUMBACH M. M., SHEPARD T. H., 1972. The ontogenesis of human fetal hormones. I. Growth hormone and insulin. J. clin. Invest., 51, 3080-3083.

KHORRAM O., DE PALATIS L. R., McCANN S. M., 1983. Development of hypothalamic control of growth hormone secretion in the rat. Endocrinology, 113, 720-728.

KINGSBURY B. F., ROEMER F. J., 1940. The development of the hypophysis of the dog. Am. J. Anat., 66, 449.481. 
LEMIRE R. J., BECKWITH J. B., WARKANY J., 1978. Anencephaly. Raven Press, N. Y.

LI J. Y., DUBOIS M. P., DUBOIS P. M., 1977. Somatotrophs in the human fetal anterior pituitary An electron microscopic immunochemical study. Cell Tissue Res., 181, 545-552.

LIDOV H. G. W., MOLLIVIER M. E., 1982. Immunohistochemical study of the development of serotoninergic neurons in the rat CNS. Brain Res. Bull., 9, 559-604.

LIOTTA A., OSATHANONDH R., RYAN K., KRIEGER D. T., 1977. Presence of corticotropin in human placenta : demonstration of in vitro synthesis. Endocrinology, 101, 1552-1558.

LUBBERHUIZEN H. N., 1931. Die Entwicklung der Hypophysis cerebri bein Schaft (Ovis aries). Z. Anat. Entwicklungsgech., 96, 1-28.

MACARON C., FREINKEL N., BRENER J., 1976. Gonadotropin-releasing hormone (GnRH) stimulates human chorionic gonadotropin (HCG) release in trophoblastic disease. Clin. Res., 24, $274 \mathrm{~A}$.

MAGRE S., DUPOUY J. P., 1973. Etude cytochimique de l'adenohypophyse du Rat. Arch. Anat. micr. Morphol. exp., 62, 217-232.

MAILLARD M., 1963. Origine des grains de sécrétion dans les cellules de l'antéhypophyse embryonnaire du rat : rôle de l'appareil de Golgi. J. Microsc., 2, 81-89.

MARCOVITZ S., GOODYER C. G., GUYDA H., GARDINER R. J., HARDY J., 1982. Comparative study of human fetal, normal adult, and somatotropic adenoma pituitary function in tissue culture. J. clin. Endocrinol. Metab., 54, 6-16.

MARTI-HENNEBERG C., GLUCKMAN P. D., KAPLAN S. L., GRUMBACH M. M., 1980. Hormone ontogeny in the ovine fetus. XI. The serotoninergic regulation of growth hormone and prolactin secretion. Endocrinology, 107, 1273-1277.

MATSUZAKI F., IRIE M., SHIZUME K., 1971. Growth hormone in human fetal pituitary gland and cord blood. J. clin. Endocrinol. Metab., 33. 908-911.

MITNIK M., CHIEFFO V., GIBBONS J. M., HANLEY K. J., 1974. Isolation of TRF and LRH from human amniotic fluid and their in vitro binding to human chorio-amniotic membranes. Proc. 56 th Meet. Endocr. Soc., Atlanta. Abstr., p. 126.

MONROE B. G., PAULL W. K., 1974. Ultrastructural changes in the hypothalamus during development and hypothalamic activity : the median eminence. Progr. Brain Res., 41, 186-212.

MOSES A. C., NISSLEY S. P., SHORT P. A., RECHLER M. M., WHITE R. M., KNIGHT A. B., HIGA O. Z., 1980. Increased levels of multiplication-stimulating activity, an insulin-like growth factor, in fetal rat serum. Proc. nat. Acad. Sci., 77, 3649-3653.

MUELLER P. L., PICTET R. L., KAPLAN S. L., GRUMBACH M. M., 1978. The ontogenesis of somatostatin in the fetal rat and sheep. Clin. Res., 26, 190 (Abstr.).

NELSON W. O., 1933. Studies on the anterior hypophysis. Am. J. Anat., 52, 307-324.

NOBIN A., BJORKLUND A., 1973. Topography of the monoamine neuron systems in the human brain as revealed in fetuses. Acta physiol. scand., suppl. 388, 1-40.

NOGIMORI T., ALEX S., BAKER S., EMERSON C. H., 1985. Thyrotropin-releasing hormone metabolism and extraction by the perfused guinea pig placenta. Endocrinologv, 117, 565-570.

OHMURA E., JANSEN A., CHERNICK V., WINTER J., FRIESEN H. G., RIVIER J., VALE W., 1984. Human pancreatic growth hormone releasing factor (hpGRF-1-40) stimulates $\mathrm{GH}$ release in the ovine fetus. Endocrinology, 114, 299-301.

OJEDA S. P., JAMESON H. E., 1977. Developmental patterns of plasma and pituitary growth hormone $(\mathrm{GH})$ in the female rat. Endocrinology, 100, 881-889.

OLIVER C., ESKAY R. L, PORTER J. C., 1980. Developmental changes in brain TRH and in plasma and pituitary TSH and prolactin levels in rat. Biol. Neonate, 37, 145-152.

OLIVER C., GIRAUD P., LISSITSKY C., CONTE-DEVOLX B., GILLIOZ P., 1981. Influence of thyrotropin-releasing hormone on the secretion of thyrotropin in neonatal rat. Endocrinology, 108, 179-183.

OLIVER C., GIRAUD P., LISSITZKY J. C., COTE J., BOUDOURESQUE F., GILLIOZ P., CONTEDEVOLX B., 1982. Influence of endogenous somatostatin on growth hormone and thyrotropin secretion in neonatal rats. Endocrinology, 110, 1018-1022.

OPPENHEIMER C. L., PESSIN J. E., MASSAGUE J., GITOMER W., CZECH M. P., 1983. Insulin action rapidly modulates the apparent affinity of the insulin-like growth factor II receptor. $J$. biol. Chem., 258, 4824-4830.

O'RAHILLY R., GARDNER E., 1971. The timing and sequence of events in the development of the 
human nervous system during the embryonic period proper. Z. Anat. Entwiklung Gesch., 134. 1-23.

PAULIN C., BARRY J., DUBOIS M. P., DUBOIS P. M., 1977. Immunofluorescence study of LHRH producing cells in human fetal hypothalamus. Cell Tissue Res., 182, 341-345.

PAULIN C., LI J. Y., BEEGEOT M., DUBOIS P. M., 1976. La somatostatine et la fonction somatotrope anthypophysaire chez le foetus humain, 129-138. In R. MORNEX, Coll. Actions thématiques 22 et 35 , Neuroendocrinologie, INSERM, Paris.

PAVLOVA E. B., PRONINA T. S., SKEBELSKAYA Y. B., 1968. Histostructure of adenohypophysis of human fetuses and contents of somatotrophic and adrenocorticotropic hormones. Gen. comp. Endocrinol., 10, 269-278.

PELLETIER G., GUY J., DUBE D., LECLERC R., BLANCHARD L., MARCHETTI B., 1982. Ontogenesis of hypothalamic factors controlling pituitary secretion. In SAEZ J. M., BERTRAND J., DUCHARME J. R., COLLU R., Ontogenesis in the endocrine system, INSERM, Paris, 109, 103-116.

PILGRIM C., 1974. Histochemical differentiation of hypothalamic areas. Progr. Brain Res., 41, 97-123.

REPPERTS S. M., SCHWARTZ W. J., 1984. Prenatal development of a functional circadian clock. In F. LABRIE, L. PROULX, Endocrinology, Elsevier. Int. Congr. Series 655, 701-704.

RICHMAN R. A., BENEDICT M. R., FLORINI J. R., TOLY B. A., 1985. Hormonal regulation of somatomedin secretion by fetal rat hepatocytes in primary culture. Endorcinology, 116, 180188.

RIEUTORT M., 1974. Pituitary content and plasma levels of growth hormone in fetal and weanling rats. J. Endocrinol., 60, 261-268.

RIEUTORT M., 1981. Ontogenetic development of the inhibition of growth hormone release by somatostatin in the rat: in vivo and in vitro (perifusion) study. J. Endocrinol., 89. 355-363.

RIEUTORT M., JOST A., 1976. Growth hormone in encephalectomized rat fetuses, with comments on the effects of anesthetics. Endocrinology, 98, 1123-1129.

RUSAK B., ZUCKER I., 1979. Neural regulation of circadian rhythms. Physiol. Rev. 59, 449-526.

SCHECHTER J., 1970. A light and electron microscopic study of Rathke's pouch in fetal rabbits. Gen. comp. Endocrinol., 14, 53-68.

SCHECHTER, J., 1971. The cytodifferentiation of rabbit pars distalis, an electron microscope study. Gen. comp. Endocrinol., 16, 1-14.

SETALO G., NAKANE P. K., 1972. Studies on the functional differentiation of cells in fetal anterior pituitary glands of rats with peroxidase-labeled antibody method. Anat. Rec., 172, 403-406.

SETALO G., NAKANE P. K., 1976. Functional differentiation of the fetal anterior pituitary cells in the rat. Endocr. exp., 10, 155-166.

SHAMBAUCH G. E. III, KUBEK M., WILBER J. F., 1983. Characterization of rat placental TRHlike material and the ontogeny of placental and fetal brain TRH. Placenta, 4, 329-338.

SIMPSON E. R., McDONALD P. C., 1981. Endocrine physiology of the placenta. Ann. Rev. Physiol., 43, 163-188.

SPENCER G. S. C., GARSSEN G. J., COLENBRANDER B., MEIJER J. C., 1985. Effect of somatostatin and thyrotropin-releasing hormone on the levels of growth hormone in the circulation of the chronically catheterized pig fetus in utero. J. Endocrinol, 106, 121-124.

STOKES H., BODA J. M., 1968. Immunofluorescent localization of growth hormone and prolactin in the adenohypophysis of fetal sheep. Endocrinology, 83, 1362-1367.

STRBAK V., JURCOVICOVA J., VIGAS M., 1985. Maturation of the inhibitory response of growth hormone secretion to ether stress in postnatal rat. Neuroendocrinologv, 40, 377-380.

STROSSER M. Th., MIALHE P., 1975. Growth hormone secretion in the rat as a function of age. Horm. Metab. Res., 7, 275-278.

SVALANDER C., 1974. Ultrastructure of the fetal rat adenohypophysis. Acta endocrinol., 76, suppl. $188,1-113$.

SZABO K., CZANYI K., 1982. The vascular architecture of the developing-pituitary-median eminence complex in the rat. Cell Tissue Res., 224, 563-577.

TERNEBY U. K., 1972. The development of the hypophysial vascular system in the rabbit, with particular regard to the primary plexus and the portal vessels. $J$. neurovisc. Rel., 32, 311-335.

THLIVERIS J. A., CURRIE R. W., 1980. Observations on the hypothalamo-hypophyseal portal vasculature in the developing human fetus. Am. J. Anat., 157, 441-444. 
TONOOKA N., GREER M. A., 1978. Evidence that control of fetal thyrotropin secretion is inclependent of both the fetal and maternal hypothalamus. Endocrinology, 102, 852-856.

VAN-WYK J. J., GRAVES D. R., CASELLA S. J., JACOBS S., 1985. Evidence from monoclonal studies that insulin stimulates DNA synthesis through the type I somatomedin receptor. The Endoc. Soc., 67th annu. Meet., June 19-21, Baltimore, Abs. 180.

VITUMS A., 1977. Development of the equine hypophysis cerebri; with a special reference to its blood supply. ZentralbI, Veterinaermed, 6, 119-135.

VON MUHLENDAHL K. E., PACHALY J., SCHMIDT-GOLLNITZER M., 1976. Lack of correlation between clinical data and growth hormone concentration in cord blood. Biol. Neonate, 29, 281-285.

WALKER P., DUSSAULT J. H., ALVARADO-URBINA G., DUPONT A., 1977. The development of the hypothalamo-pituitary axis in the neonatal rat : hypothalamic somatostatin and pituitary and serum growth hormone concentration. Endocrinology, 101, 782-787.

WALLACE J. A., LAUDER J. M., 1983. Development of the serotonergic system in the rat embryo: an immunocytochemical study. Brain Res. Bull., 10, 459-479.

WATANABE Y. G., DAIKOKU S., 1976. Immunohistochemical study on adenohypophyseal primordia in organ culture. Cell Tissue Res., 166, 407-412.

WEHRENBERG W. B., 1986. The role of growth hormone-releasing factor and somatostatin on somatic growth in rats. Endocrinology, 118, 489-494.

YOSHIMURA $F_{1}$, HARUMIYA K., KIYAMA H., 1970. Light and electron microscopic studies of the cytogenesis of anterior pituitary cells in perinatal rats in reference to the development of target organs. Arch. Histol. Jap., 31, 333-369.

YOUNGBLOOD W. W., HUMM J., L!PTON M. A., KIZER J. S., 1980. Thyrotropin-releasing hormone like bioactivity in the placenta : evidence for the existence of substances other than pyro-Glu-His-Pro $\mathrm{NH}_{2}$ (TRH) capable of stimulating pituitary thyrotropin release. Endocrinology, 106, 541-546. 